\title{
PROTEIN BAITS, VOLATILE COMPOUNDS AND IRRADIATION INFLUENCE THE EXPRESSION PROFILES OF ODORANT- BINDING PROTEIN GENES IN BACTROCERA DORSALIS (DIPTERA: TEPHRITIDAE)
}

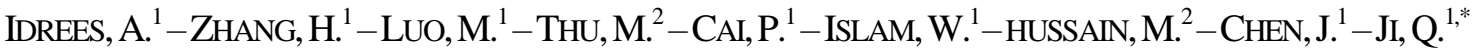 \\ ${ }^{1}$ Institute of Beneficial Insects, Plant Protection College, Fujian Agriculture and Forestry \\ University, Fuzhou, Fujian, 350002, China \\ ${ }^{2}$ College of Horticulture, Fujian Agriculture and Forestry University \\ Fuzhou, Fujian, 350002, China \\ (phone:+86-0-59-183-789-420; fax:+86-0-59-183-789-421) \\ *Corresponding author \\ e-mail: jiqinge@yeah.net; phone:+86-0-59-183-789-420; fax:+86-0-59-183-789-421
}

(Received $4^{\text {th }}$ Jul 2017; accepted $18^{\text {th }}$ Oct 2017)

\begin{abstract}
Chemical communication in insects is based on odorant-binding proteins (OBPs), but the molecular mechanisms by which these OBPs allow the perception of volatiles and host chemicals remain unclear. Bactrocera dorsalis (Hendel) is one of the most economically important pests of fruits and vegetables. In this study, the OBPs of $B$. dorsalis (BdorOBPs) were evaluated in response to different attractive protein baits, brewer's yeast volatile compounds and irradiation. The expression of 10 genes encoding OBPs in the antennae of $B$. dorsalis during three adult physiological life stages (pre-mating, post-mating and post-oviposition) were analysed. All selected BdorOBP genes were found to contain one conserved pheromone-binding protein/general-odorant-binding protein domain (PBP-GOBP), except OBP8, which contained two of these conserved domains. An unrooted phylogenetic tree was constructed to show the relationships among these 10 BdorOBPs and the OBPs belonging to the same OBP family in other insects. We found significantly different transcript expression profiles in each OBP gene at each stage in response to different treatments; these results revealed that OBP2 expression was significantly increased in response to baits at each adult physiological stage, while OBP2, OBP5 and OBP1 were highly expressed in response to combined-volatiles treatment at all tested physiological stages. OBP3, OBP5 and OBP10 showed high expression in response to irradiation at all tested physiological stages. Thus, we infer that protein baits, brewer's yeast volatiles and irradiation significantly influence the transcript levels of OBP genes, which may act in olfactory perception.
\end{abstract}

Keywords: transcript analysis, irradiation, brewer's yeast volatiles, oriental fruit fly, insect olfaction

\section{Introduction}

Bactrocera dorsalis is the most devastating pest in several Asian countries including China. This fruit fly was reported for the first time in Taiwan in 1912 (Hardy, 1973). B. dorsalis is a serious quarantine pest throughout the world, resulting in severe financial damage to multiple crops that are among its 270 host plant species (Clarke et al., 2005; Chen et al., 2008; Leblanc et al., 2013; Vargas et al., 2015). The extensive damage caused by this particular pest worldwide raises serious concerns about crop yields due to the high resistance of this pest to chemical insecticides (Hu et al., 2014). A variety of methods such as the sterile insect technique, mass trapping, protein bait spraying and male annihilation have been developed to suppress or eradicate $B$. dorsalis and related species throughout Asia and the Pacific regions (Jessup et al., 2007; Mau et al., 2007; Piñero et al., 2009a; Piñero et al., 2009b; Vargas et al., 2015). 
Chemoreception is an important characteristic in the selection of host plants by phytophagous insects. Odorant-binding proteins (OBPs) present in the olfactory system play an essential role in sensitivity to odorants, such as pheromones and host chemicals (Zheng et al., 2013). The olfactory system in insects consists of three central types of proteins: OBPs, odorant receptors (ORs) and odorant-degrading esterases (ODEs) (Justice et al., 2003). Among these proteins, OBPs frequently function in the first step of odour perception. The process of chemical communication in both insects and vertebrates is performed by OBPs (Tegoni et al., 2004; Dahanukar et al., 2005; de Bruyne and Baker, 2008). Insects possess a notably complex and cosmopolitan olfactory system, through which they are potentially able to detect a variety of external volatile compounds including plant volatiles and gender-specific mating pheromones (Hsu et al., 2012; Zheng et al., 2012b; Papadopoulos et al., 2013; Zheng et al., 2013; Liu et al., 2015). Insect OBPs may represent an important molecular target in the application of eco-friendly pest management approaches, as they are proposed to be involved in olfactory cue discrimination due to their intense expression in antennal sensillum lymph (Qiao et al., 2009; He et al., 2011; Sun et al., 2013a, 2014; Pelosi et al., 2014). Insects usually use their antennae as olfactory organs in order to detect the odours of plants, fruits and chemicals in the outside environment (Sun et al., 2016a).

Conversely, many studies have shown that the expression of OBPs is not limited to the olfactory organs; thus, their physiological functions may be broader and more difficult to understand than expected (Park et al., 2000; Foret and Maleszka, 2006; Li et al., 2015; Sun et al., 2012; Yuan et al., 2015). To determine whether the OBPs in the antennae of Bactrocera species respond to volatiles, the present study focuses on the expression patterns of 10 OBPs in the antennae of B. dorsalis (BdorOBPs) in response to protein baits, volatiles and irradiation. Insect OBPs, which are specifically or primarily expressed in the antennae, have been proposed to play roles in olfactory function in response to different kinds of attractive host plant volatiles (Zhou, 2010; Leal, 2013). Previously, an electroantennogram (EAG) was employed to record the electrophysiological responses of the antennae of $B$. dorsalis to spent brewer's yeast volatiles, alcohols, aldehyde alcohols and aldehydes to determine the olfactory response of the antennae towards chemical odours. The results of that study revealed that volatiles could potentially evoke higher relative EAG values in the antennae of $B$. dorsalis during three adult physiological stages (Jang and Light, 1991; Du et al., 2015). A total of 20 BdorOBP genes have been identified from the $B$. dorsalis transcriptome; however, only 10 BdorOBPs have been characterized (Zheng et al., 2012b, 2013).

$B$. dorsalis transcriptomes and expression analysis in different tissues have been analysed, but the study of the molecular basis of olfaction in response to different stimuli is still in its initial stages (Shen et al., 2011a; Geib et al., 2014). In the studies mentioned above, scientists have reported the EAG response of $B$. dorsalis to different chemical odours and identified OBP genes that are responsible for the first step in the perception of chemical odours. However, few studies have evaluated the expression profiles of the different OBP genes of an insect pest in response to different volatiles, particularly different baits, spent brewer's yeast volatiles and irradiation. Hence, there is a pressing need to study the influence of different kind of volatiles on the expression levels of OBP genes in the antennae of $B$. dorsalis.

Therefore, the current study was based on the hypothesis that different protein baits, spent brewer's yeast volatiles and irradiation would influence the expression levels of OBP genes at three adult physiological stages in the antennae of $B$. dorsalis. The objective of the study was 
to evaluate the effects of different protein baits, spent brewer's yeast volatiles and the application of radiation on the transcript levels of the 10 previously identified BdorOBP genes in the antennae of $B$. dorsalis during the pre-mating, post-mating and post-oviposition stages. The findings of the present study may provide knowledge of the molecular mechanisms of action of the variety of OBPs present in the antennae of $B$. dorsalis and potentially suggest novel tactics for the suppression of $B$. dorsalis. A greater understanding of the mechanisms underlying OBP function may allow the invention of novel control methods to disrupt these internal physical mechanisms, perhaps by influencing the gene expression profiles of OBP genes and thus support novel strategies for long-term effective management of tephritid fruit fly species.

\section{Material and Methods}

\section{Insect rearing}

The insects were reared at the Institute of Beneficial Insects, Fujian Agriculture and Forestry University, Fuzhou, Fujian Province, China, under controlled conditions $\left(26 \pm 1^{\circ} \mathrm{C}\right.$; $60-70 \% \mathrm{RH} ; 12 \mathrm{hr}$ light/12 hr dark photoperiod) for approximately 60 generations. The eggs of $B$. dorsalis were collected using a homemade ovipositional bottle including artificial juice and then were placed on a single layer of wet tissue paper in a larval diet tray. The adult flies were reared on artificial diet prepared with yeast extract and sugar in accordance with the procedures described by (Spencer and Fujita, 1998).

\section{Experimental materials}

Proteins were prepared at the Institute of Beneficial Insects at Fujian Agriculture and Forestry University, Fuzhou, Fujian Province, China, while torula yeast was obtained from Baifude Biotechnology, Wuxi, Jiangsu. Spent brewer's yeast volatile compounds phenyl acetate, ethyl caprylate, and octyl acetate (analytical reagents) were purchased from Solarbio Pvt. Ltd., Shanghai, while paraffin oil (100\%) was obtained from Acros Organics (Geel, Belgium).

\section{Experimental methods}

Protein baits and volatile regulation for female and irradiation application method for male B. dorsalis

This experiment was planned to evaluate the effects of protein baits and torula yeast on the transcript expression of 10 previously identified full-length cDNAs of OBP genes in the antennae of $B$. dorsalis at different adult life stages: pre-mating (2-3 days), post-mating (7-9 days) and post-oviposition (11-13 days). A total of one hundred (100) mature females were released in a $33 \mathrm{~cm} \times 33 \mathrm{~cm} \times 33 \mathrm{~cm}$ cage and starved for $12 \mathrm{hrs}$ overnight prior to feeding using protein bait and torula yeast. The insects were treated with two different protein baits (1:10 dilution with paraffin oil), whereas the control group was treated with an equal volume of normal protein diet (Zheng et al., 2012b). Three spent brewer's yeast volatile compounds and their blend mixtures were prepared: $15 \%$ paraffin oil (Control, Ck), 15\% (75 $\mu 1$ phenyl acetate $+425 \mu 1$ paraffin oil), $25 \%$ (125 $\mu$ l ethyl caprylate $+375 \mu$ l paraffin oil), 15\% (75 $\mu$ l octyl acetate $+425 \mu 1$ paraffin oil) and a mixture of all compounds ( $15 \%$ phenyl acetate $+25 \%$ ethyl caprylate $+15 \%$ octyl acetate) (MIX). Treatments were denoted by Ck: control, PA: phenyl acetate, EC: ethyl caprylate, OA: octyl acetate, MIX: mixture of three volatile compounds (PA + EC 
+ OA). B. dorsalis was irradiated with ${ }^{137} \mathrm{Cs}$ at 100 Gy for 1,2 and 3 days at the pupal stage, and kept at $25 \pm 1{ }^{\circ} \mathrm{C}, \mathrm{RH} 65 \% \pm 5 \%$, and $\mathrm{L}: \mathrm{D}=12: 12$ after adult emergence for 1 day, 6 days and 8 days. Afterwards, 100 infertile males were collected on each day, and their antennae were dissected.

\section{$R N A$ isolation and $q R T-P C R$ analysis}

Adult tissue samples were dissected from 500 individuals. The total RNA for each group was extracted from the antennae of $B$. dorsalis at 2-3 days (pre-mating stage), 7-9 days (postmating stage) and 11-13 days (post-oviposition stage) by using a Tiangen RNeasy Kit. Two micrograms of total RNA was reverse-transcribed by the PrimeScript 1st strand cDNA Synthesis Kit (TaKaRa, Japan) using the oligo dT primer. qRT-PCR was conducted to detect the expression of genes encoding OBPs by using gene-specific primers (Table 1), Top Green qPCR Super Mix (Transgen, China) and a real-time thermocycler (Bio-Rad, Hercules, CA, USA) following the manufacturer's instructions. For internal standardization, $\beta$-Actin was amplified. Prior to starting the gene expression analysis, the PCR efficiency of the genes was validated. The qRT-PCR was performed in a volume of $20 \mu \mathrm{l}$ containing $10 \mu \mathrm{l}$ of $2 \times$ TransStart ${ }^{\circledR}$ Top Green qPCR Super Mix, $2 \mu \mathrm{cDNA}$ (1:100 diluted), $0.8 \mu \mathrm{l}$ of each (forward and reverse) primer and $6.4 \mu \mathrm{l}$ nuclease-free $\mathrm{H}_{2} \mathrm{O}$. The qRT-PCR programme was: $94^{\circ} \mathrm{C}$ for $30 \mathrm{~s}$ followed by 40 cycles at $94^{\circ} \mathrm{C}$ for $15 \mathrm{~s}, 60^{\circ} \mathrm{C}$ for $30 \mathrm{~s}$, and $72^{\circ} \mathrm{C}$ for $10 \mathrm{~s}$. The melting curve was analysed for the PCR products to confirm the presence of a single amplified fragment at the end of each PCR. The $\beta$-actin gene was used as a housekeeping gene to standardize the qRT-PCR results. A relative quantitative computing method ( $2^{-\Delta \Lambda C t}$ method) was used to compute gene expression (Livak and Schmittgen, 2001). Three biological replicates were performed.

Table 1. Primers used for gene expression detection of BdorOBPs by qRT-PCR

\begin{tabular}{c|c}
\hline Primer name & $\left(\mathbf{5}^{\prime} \rightarrow \mathbf{3}\right)$ Nucleotide sequence \\
\hline BdorOBP1RTF & GGCGAGCGTTGTTCCAG \\
BdorOBP1RTR & ATCGGCACCAGCACTTCC \\
BdorOBP2RTF & TTCTTCGCTGTTGCTGTTT \\
BdorOBP2RTR & AGAAGCATTTGACTTTGCCATC \\
BdorOBP3RTF & TTGACCGAGGAGCAGAAAC \\
BdorOBP3RTR & TTGACTTTGCCATCGACTT \\
BdorOBP4RTF & GAACTTCTTCGCTGTTGCTG \\
BdorOBP4RTR & CTTGACTGTCATCGCCTTTG \\
BdorOBP5RTF & GGATTCCACGACTTCATTGAG \\
BdorOBP5RTR & CTTTAGCCGCATCAGGTTTG \\
BdorOBP6RTF & ACGAAGCCAAAGTCACGG \\
BdorOBP6RTR & GCATCAAAGACGCCATCC \\
BdorOBP7RTF & GTTGCAGGCAAATTCCAGAT \\
BdorOBP7RTR & AGAGTCCCATTCGCTCCACA \\
BdorOBP8RTF & ACGGCTAAGGCTCTAAAGAACC \\
BdorOBP8RTR & AAACCACTTGTGACGCTTCG \\
BdorOBP9RTF & GCGATGCCGACCATGATGAC \\
BdorOBP9RTR & ACCACCACGATAAGCCCACT \\
BdorOBP10RTF & GTGTCTTCTGCGAACATGAGG \\
BdorOBP10RTR & CACTTGTGACCACGATAGGC \\
$\beta$-actinRTF & CTCGTCCAACCGTTCATACC \\
$\beta$-actinRTR & CTGACCTGCCCACTGAAGTT \\
\hline
\end{tabular}




\section{Statistical analysis}

Statistical analysis was performed using the SPSS 17.0 software package (IBM Corporation, Somers, NY, USA) to calculate Dunnett's test for comparing control with other treatments (SPSS for Windows, Version 16.0, 2007).

\section{Results}

\section{Identification of PBP-GOBP domains in BdorOBP genes}

All 10 BdorOBP genes were found to contain one conserved PBP-GOBP domain, except for OBP8, which, remarkably, contained two such conserved domains. In the OBP family, the amino acid sequence residues of each BdorOBP gene were green when compared with the hidden Markov model (HMM), which indicates a very high-quality hit. BdorOBP conserved domains are presented in (Figure 1), while the BdorOBP genes along with their accession numbers are presented in (Table 2). The PBP-GOBP conserved domain was identified by using the Pfam database (version 31.0) (Finn et al., 2016).

\section{$<$ BdorOBP1|PCI domain |}

NADYEEKTEDDFLSAGERCFQRERLAASYQRRFDNFDYPDEEPV QRYVHCIWTELKLWNDRTGFNVEHIAALYRDKANTEVLVPILSDC NRNAQNEPTLKWCYRAFKCVLNS

$<$ BdorOBP2|PCI domain

YPPPELLKELQPVHDSCVAKTGVTEEAIKEFSDGDVHEDELLKCYM YCVFEETDVLHEDGEVHLEKILDKLPESMHVIALHMGKKCLYPKGD NKCERAFWLHR

$<$ BdorOBP3|PCI domain |

GKLTEEQKQKVHAAAAECFKETGASEDAVRALLKGDDSQVDGKVK CFAKCTLGKLDLLQNGKVNEEKVQNILGKLIGEEKAKAAQAKCNGL KGTDECDTAYQIRQCYAAG

$<$ BdorOBP4|PCI domain | EGMLTPEQIQKVHTLSNECLKETGASEDAIRALIKGDDSQVDGK VKCYAQCMLVKLGYVENGKVNEEKVQNILGKLIGEEKAKATQ AKCNGLKGTDECDTAYQIRQCYSAG

$<$ BdorOBP5|PCI domain |

PHDPEMRGYIEDCNKEHNVSPKDFHDFIEGKLTTVPENMKCSSQC IMVKQGIMDESGNFKPDAAKAKMKEDKLVAAVDECKDLSGSTPC DTAFKITSCMLSK

\section{$<$ BdorOBP6|PCI domain |}

AVCQLPADLEKFHKACMDEAKVTDEQMRQFFQNGMKASDATENI KCQMKCMMQKQGIWKDGVFDADAKIKELVQNPKFKGKEARTNK
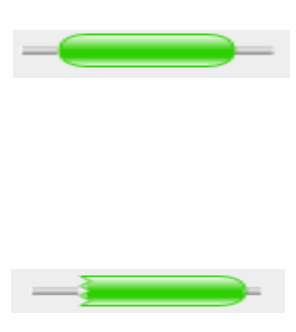

$<$ BdorOBP7|PCI domain |

GKFQIRTAQDALDAHEACHEEYRVPEDIYQKFLNYEFPAHKRTNCY VKCFVERMGLFTEEKGFDEKAIIAQFTAKSSKNLAKVSHGLEKCLDH NEHDSDTCTWANRVFSCWIS 


\author{
$<$ BdorOBP8|PCI domain | \\ ATTKAAEQDVDSDTEILRKCLREVGSKDLVGELQKVARYSKW \\ TSEEVPCFTRCLASMKHWFDADESKWNKQQIADDLGADMYN \\ YCRYELDRYNEDSCEFAYTGLRCLKQA
}

TLETYKNIVSCASELNVTMKELQKYAAFPTKEVVPCLFQCLAE KMNFYTPTYEWNLDNWVQAFGPMRQDRTASNVCKVSAEHM KTRDKCEWMYEEYNCLER

$<$ BdorOBP9|PCI domain | SAEYVVKNEENLQQYRRECATELKVPAEHIEQFRKWQFPNDA VTQCYLKCVFEKFGLFDAVTGFNVEHIHQQLQGAEVAPPGDA DHDDVVHDKIAACVDTNEQGSNACEWAYRGGVCFIKE

$<$ BdorOBP10 | PCI domain | DEEWVPKNVAQIKAIRQECIKDFPLSEEYIQKMKNFEYPDEEPVR KYLLCTAKKLGVFCEHEGYHADRVAKQFKMDLDEAEVIAIAEG CADKNVEGSSADVWAYRGHKCVMAS
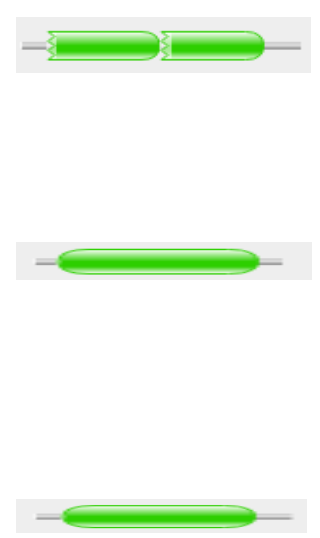

Figure 1. BdorOBPs contain one conserved PBP-GOBP domain, which is a typical characteristic of OBPs. All BdorOBP genes contain one conserved PBP-GOBP domain, except $O B P 8$, which contains 2 of these conserved domains. The PBP-GOBP domains were identified by using the Pfam database (version 31.0), and the amino acid sequence residues of each BdorOBP gene were green when compared with the hidden Markov model (HMM), which indicates a very high-quality hit.

Table 2. BdorOBP genes with their accession numbers and nucleotide lengths

\begin{tabular}{c|c|c}
\hline Gene name & Accession number & Length \\
\hline BdorOBP1 & KC559112 & 159 \\
BdorOBP2 & KC559113 & 138 \\
BdorOBP3 & $K C 559114$ & 146 \\
BdorOBP4 & $K C 559115$ & 136 \\
BdorOBP5 & $K C 559116$ & 130 \\
BdorOBP6 & $K C 559117$ & 104 \\
BdorOBP7 & $K C 559118$ & 142 \\
BdorOBP8 & $K C 559119$ & 274 \\
BdorOBP9 & $K C 559120$ & 148 \\
BdorOBP10 & $K C 559121$ & 149 \\
\hline
\end{tabular}

\section{Phylogenetic analysis of insect $\mathrm{OBP}$}

A phylogenetic tree was generated by obtaining the amino acid sequences of the ten putative OBPs of $B$. dorsalis from the work of (Zheng et al., 2013). The phylogenetic tree was built to demonstrate the relationships of the 10 BdorOBPs to $140 \mathrm{OBP}$ sequences from 7 different orders and families of insects that possess the same OBP family, including 20 OBP sequences from Apis mellifera (Hymenoptera: Apidae), 7 OBP sequences from Bombyx mori (Lepidoptera: Bombycidae), 30 OBP sequences 
from Drosophila pseudoobscura (Diptera: Drosophilidae), 29 OBP sequences from Halyomorpha halys (Hemiptera: Pentatomidae), 15 OBP sequences from Spodoptera litura (Lepidoptera: Noctuidae), 10 OBP sequences from Tenebrio molitor (Coleoptera: Tenebrionidae) and 44 OBP sequences from Tribolium castaneum (Coleoptera: Tenebrionidae), as shown in (Figure 2). This phylogenetic tree clarified the connections between $B$. dorsalis and other insects in the groups listed above. Amino acid sequences were used to build an unrooted phylogenetic tree using MEGA 7.0 with the pairwise deletion option under the JTT empirical amino acid substitution model (Kumar et al., 2016). Branch support was assessed by bootstrap analysis with 1000 replicates.

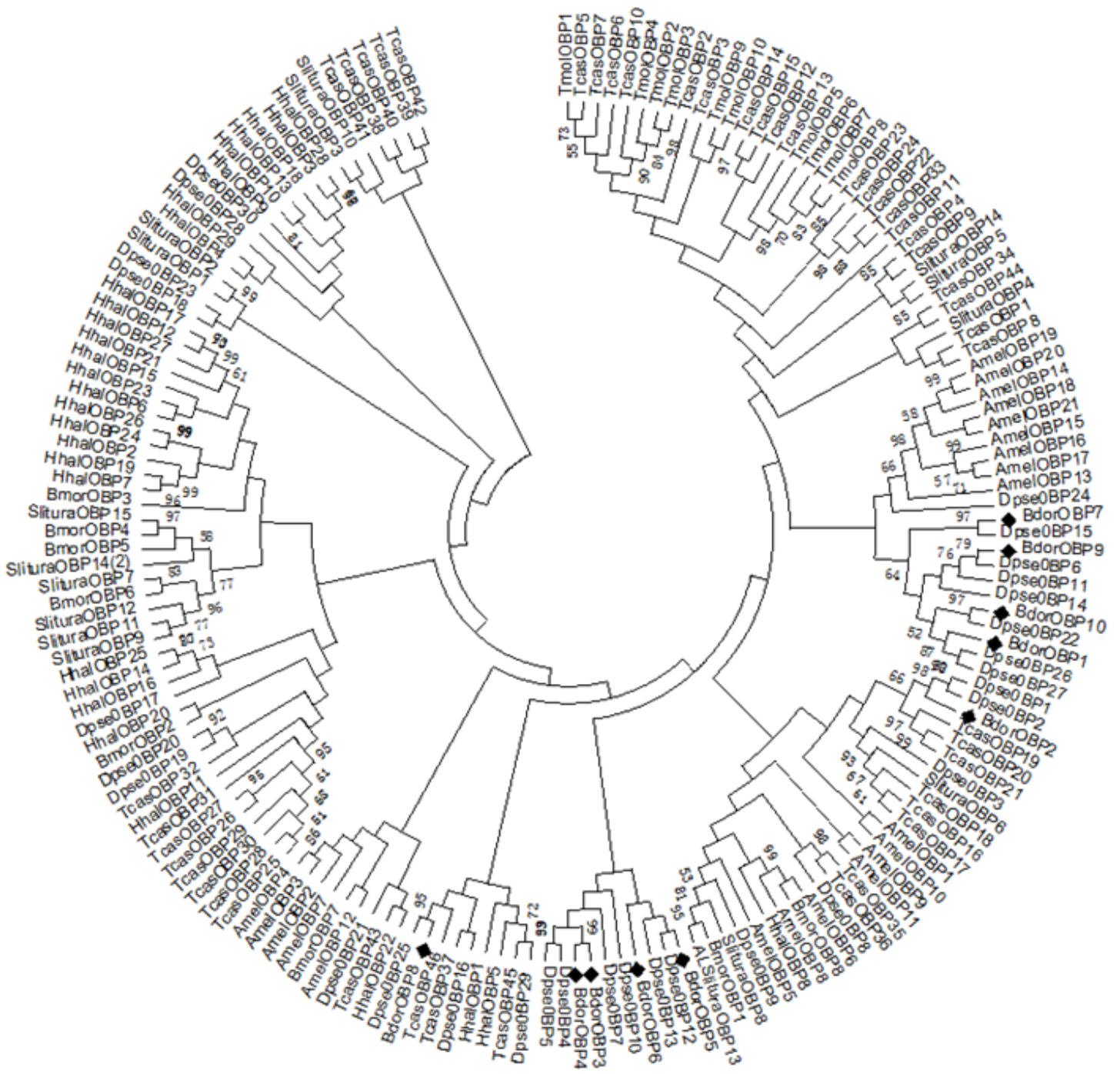

Figure 2. Unrooted phylogenetic tree of candidate OBPs from Bdor (B. dorsalis) and seven additional insect species with available amino acid sequences (Amel: Apis mellifera, Bmor: Bombyx mori, Dpse: Drosophila pseudoobscura, Hhal: Halyomorpha halys, Slitura: Spodoptera litura, Tmor: Tenebrio molitor and Tcas: Tribolium castaneum). The highly divergent signal peptides in the $N$-terminal region were removed, and a neighbour-joining tree was constructed by using MEGA 7 with the pairwise deletion option under the JTT empirical amino acid substitution model. Only bootstrap values above 50\% are shown. The black diamonds indicate the $10 \mathrm{~B}$. dorsalis OBP genes. 


\section{Effects of bait and torula yeast on BdorOBP transcript levels in females}

The qRT-PCR results revealed that all BdorOBPs showed significant changes in expression in response to protein bait and torula yeast except OBP4, OBP8 and OBP9, as shown in (Figure 3a). However, BdorOBP3 showed no significant expression in response to starvation (Figure $3 a$ ).

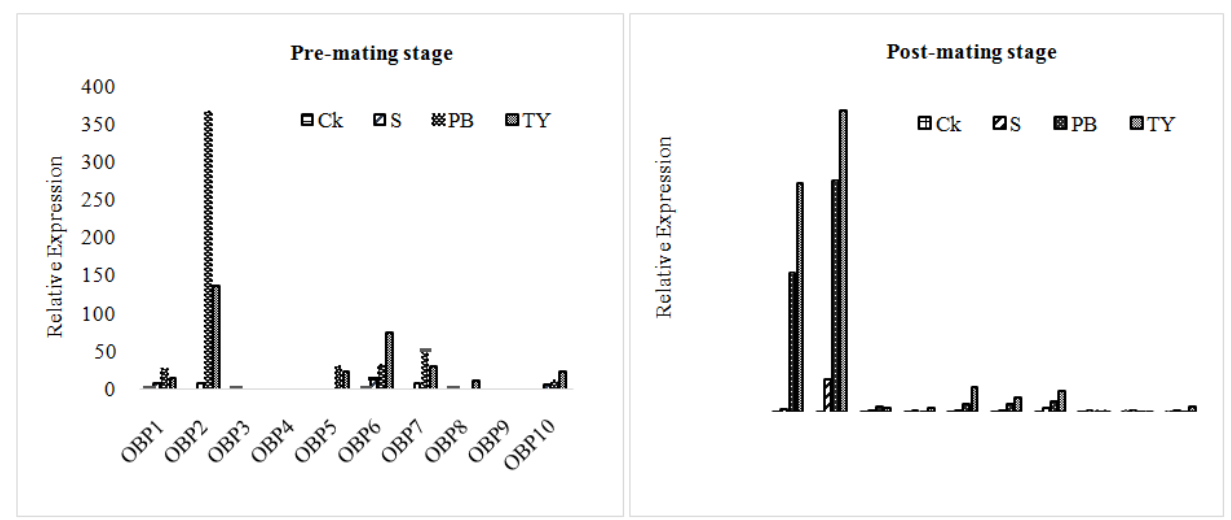

(a)

(b)

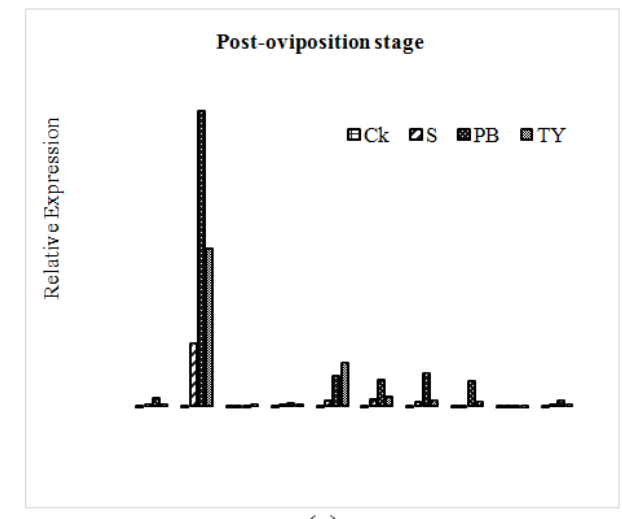

(c)

Figure 3. Transcript expression analysis by $q R T-P C R$ of the ten BdorOBP genes in the antennal tissues of mature adult females in response to protein bait and torula yeast. (a) Pre-mating stage; (b) post-mating stage; (c) post-oviposition stage. Treatments were denoted by $C k$ : control, S: starvation, PB: protein bait, TY: torula yeast. Analysis of variance (ANOVA) indicated a significant difference in the expression levels of OBPs at $p<0.05$. However, OBP3 was expressed weakly under starvation treatment, and hence, ANOVA indicated that this difference was not significant (Figure 3a). The ANOVA indicated a significant difference among the expression levels of all BdorOBPs ( $p<0.05$, Figure $3 b)$, except those of OBP9 and OBP10. However, OBP6 in response to starvation and OBP8 to starvation and protein bait were not highly expressed (Figure 4b). The ANOVA indicated a significant difference $(p<0.05)$ among the expression levels of all BdorOBPs (Figure $3 b$ ), while OBP1 in response to starvation and torula yeast; $\mathrm{OBP} 4, \mathrm{OBP} 8, \mathrm{OBP} 9$ to starvation; and $\mathrm{OBP} 10$ to starvation and torula yeast were not expressed significantly (Figure 3c).

The expression levels of OBP1, OBP2, OBP5, OBP6 and OBP7 were higher in insects given protein bait and torula yeast than those of OBP3 (Figure 3b). OBP6 and OBP8 exhibited no significant expression in response to starvation and protein bait treatments, respectively ( $p>0.05$, Dunnett's test) (Figure 3b). However, the 
transcript levels of OBP4, OBP9 and OBP10 were not altered significantly ( $p>$ 0.05 ) in response to any of the treatments (Figure $3 b$ ). Protein bait and torula yeast increased the transcript level of OBP6, while all of the remaining OBPs showed no response to protein bait and torula yeast, as shown in (Figure $3 b$ ). The expression levels of OBP1 and OBP10 were significantly increased in the antennae of $B$. dorsalis in response to starvation and torula yeast, while those of OBP8 and OBP9 showed no response to starvation. OBP4 showed no change in expression in response to the baits at any of the tested stages (Figure 3c). These results suggest that different baits may significantly alter the transcript profiles of some BdorOBPs, which may play an important role in altering the mechanism of insect olfaction.

\section{Effects of volatiles on BdorOBP transcript levels in females}

The analysis of the volatile treatments indicated relatively low expression levels in OBP5, OBP8, OBP9 and OBP10, while the remaining BdorOBPs showed significantly higher expression in all treatments, as shown in (Figure 4a).

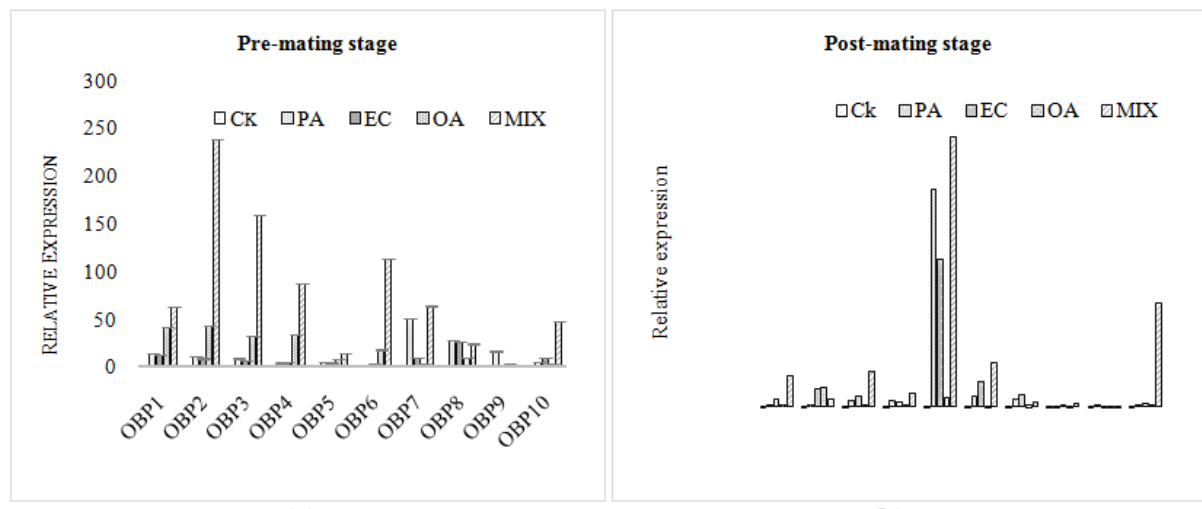

(a)

(b)

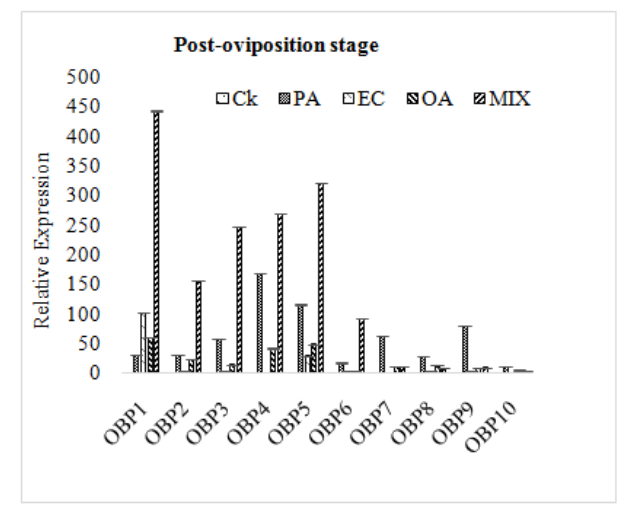

(c)

Figure 4. Transcript analysis of the ten BdorOBP genes in the antennal tissues of mature adult females in the (a) pre-mating stage; (b) post-mating stage; and (c) post-oviposition stage. Treatments were denoted by $C k$ : control, PA: phenyl acetate, EC: ethyl caprylate, OA: octyl acetate, MIX: mixture of three volatile components $(P A+E C+O A)$. Relative transcript levels were calculated using $\beta$-actin as the standard. A significant difference in the expression levels of BdorOBP genes was found by Dunnett's test $(p<0.05)$. OBP6 in response to starvation (Figure 4a), OBP8 to PA and EC (Figure 4b), OBP6 to OA, and OBP10 to PA and EC were not expressed significantly (Figure 4c). 
Interestingly, higher expression levels were observed in response to MIX and OA at all tested stages, as shown in (Figure $4 a, 4 b$ and $4 c$ ). Only OBP5 exhibited a significant expression change in response to all treatments, as shown in (Figure $4 b)$. In contrast to the lower expression levels observed in the last five BdorOBPs (OBP6-OBP10), the first five BdorOBPs (OBP1-OBP5) showed significantly increased expression in response to all treatments, particularly MIX and PA, as shown in (Figure 4b). OBP6 showed no change in expression level in response to PA and OA, as shown in (Figure 4a, 4b), while OBP8 and OBP10 also showed no change in response to PA and $\mathrm{EC}$, as shown in (Figure $4 b, 4 c$ ).

\section{Effects of irradiation on BdorOBP transcript levels in males}

The qRT-PCR results showed that the transcript levels of the BdorOBPs changed significantly at all tested stages with a ${ }^{137} \mathrm{Cs}$ irradiation dose of $100 \mathrm{~Gy}$. The expression levels of the BdorOBPs were altered significantly in response to irradiation, except those of OBP3, OBP4 and OBP7. The expression levels of OBP2, OBP5, OBP7 and OBP9 were significantly increased, as shown in (Figure 5a).

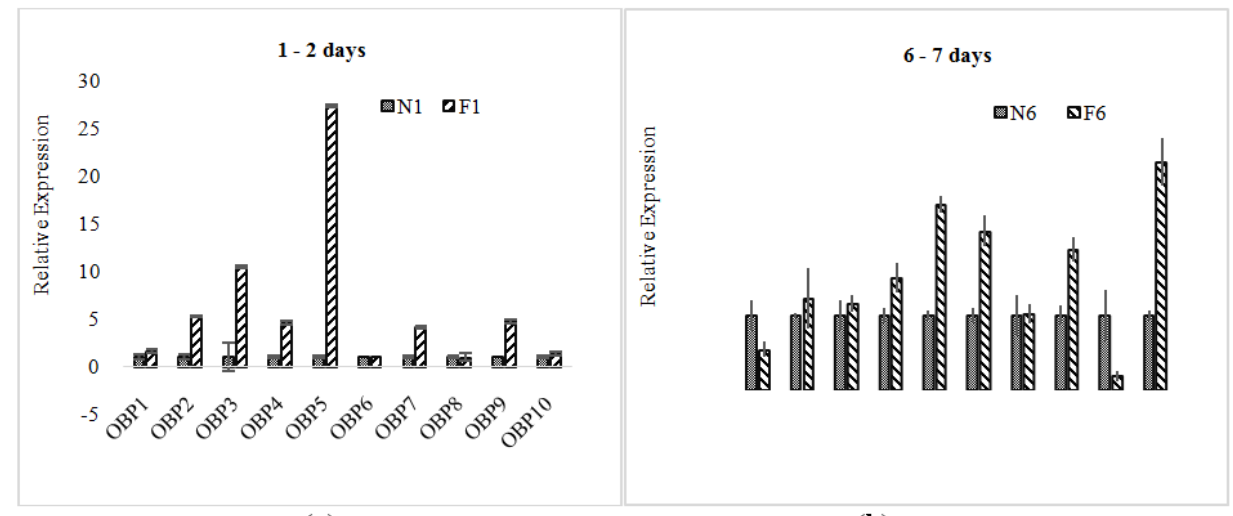

(a)

(b)

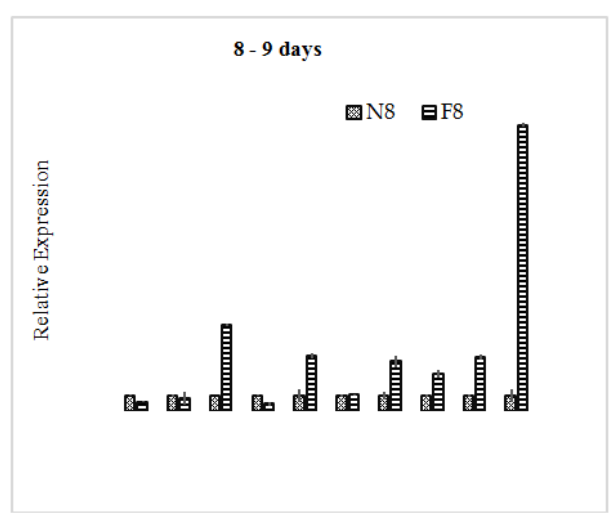

(c)

Figure 5. Transcripts of the ten BdorOBP genes in the antennal tissues of adult males at (a) 1-2 days; (b) 6-7 days; (c) 8-9 days. The treatments were denoted by $N$ : normal male and $F$ : irradiated (infertile) male. Relative transcript levels were calculated using $\beta$-actin as the standard. A significant difference among the expression levels of all BdorOBP genes was found at $p<0.05$. Low expression levels were observed in OBP3, OBP4, and OBP7 (Figure 5a), while $O B P 1$ did not show significant expression ( $p>0.05$, Figure $5 b, 5 c$ ). 
However, each OBP was significantly differentially expressed, except OBP1, which showed no change. OBP4, OBP5, OBP6, OBP8, and OBP10 exhibited particularly high expression in response to irradiation, as shown in (Figure 5b). OBP3, OBP5, OBP7, OBP8, OBP9 and OBP10 showed exceptionally abundant expression in contrast to the other OBPs, as shown in (Figure 5c). However, OBP1 showed no response to irradiation, as shown in (Figure $5 b, 5 c$ ).

\section{Discussion}

Insects essentially depend on the antennae to distinguish plant volatiles or sex pheromones in the environment (Steinbrecht, 1997); hence, we selected B. dorsalis antennae to evaluate the expression profiles of 10 BdorOBP genes in response to protein baits, brewer's yeast volatiles and irradiation. Previously published papers have reported functional studies of insect OBPs in chemoreception since their discovery in 1981, but the exact functions of insect OBPs in response to different stimuli present in the environment are still unknown (Zhou, 2010; Leal, 2013). Highly developed olfactory systems are present in phytophagous insects, allowing them to use numerous chemical stimuli to identify plant hosts (Pelosi et al., 2006). The OBPs in the olfactory signal reception pathway play an essential role in the transportation of specific odorants to ORs to stimulate insect behaviours (Pelosi et al., 2006). Hence, OBPs are thought to be directly essential for odorant perception and represent potential targets for pest control via insect attraction.

B. dorsalis antennae have a strong EAG response to spent brewer's yeast volatiles (Du et al., 2015), and the results of the present study revealed that OBP2, OBP5 and OBP1, which are present in the antennae of $B$. dorsalis, were highly expressed in response to spent brewer's yeast volatiles at all tested stages. OBPs show significant attraction responses to different volatiles (Cornelius et al., 2000; Hu et al., 2007; Liu et al., 2010; Shen et al., 2011b). Another author has reported that some volatile compounds, such as benzaldehyde, showed very weak attraction effects in all adult physiological stages of female B. dorsalis (Ling, 2013), and the results of our study also showed that some OBPs were down-regulated, perhaps due to the weak affinity of volatiles to some OBPs. A similar phenomenon was observed in the case of ester alcohols, the major constituents of aromatic odour in most fruits, which plays an essential role in the attraction behaviour of B. dorsalis (Zhang et al., 2007). Tephritidae have shown significant differences in their responses to other esters, such as beer waste yeast, hydrolysates, and volatile compounds of the other 3 kinds of esters (Du et al., 2012).

Previously, thirty-four compounds were selected to investigate the interaction of OBPs with rice plant volatiles (Lou et al., 2005; Sun et al., 2013b). Cnaphalocrocis medinalis OBP2 (CmedOBP2) showed a significant response to host volatiles, whereas CmedOBP3 showed relatively little response to these volatiles (Sun et al., 2016b); our study found that all BdorOBP genes showed low or no response to combined volatile treatments at all tested physiological stages, except the OBP2, OBP5 and OBP1 genes, which were highly expressed. Because all insects have different OBP sequences and each OBP family might perform a different function, different OBPs can display different mechanisms in response to different kinds of volatiles present in plants and fruits (Sun et al., 2016b). 
A previous report indicated that synthetic volatile compounds targeted OBP3 or OBP7, which were confirmed to be accountable for $(E)$ - $\beta$-farnesene sensitivity, and provoked significant behavioural responses in aphids (Sun et al., 2012), while the results of our studies revealed that the first five BdorOBP genes (OBP1-OBP5) were significantly expressed in response to all treatments, specifically to MIX and PA, and weak expression was observed in the last five BdorOBP genes (OBP6-OBP10) in response to volatiles.

The prerequisite for the illumination of the molecular mechanism of olfaction is the documentation of the genes accountable for volatile discrimination. Candidate olfaction genes have been recognized in numerous other species through annotation and antennal transcriptome analysis. Although $B$. dorsalis olfaction research has previously lacked transcriptome data for the classical olfactory organs, specifically the antennae, several studies of the $B$. dorsalis transcriptome have recently been conducted (Shen et al., 2011b; Zheng et al., 2012a; Geib et al., 2014). However, limited studies have investigated the effects of the volatile compounds reported for control of $B$. dorsalis on OBP gene expression profiles in $B$. dorsalis and other insect pests. In light of the present findings, the fact that $B$. dorsalis males and females were captured in greater numbers in torula yeast bait traps than in protein bait traps likely reflects a lower availability of proteinaceous food in the habitats sampled by (Siderhurst and Jang, 2010; Royer et al., 2014).

Insect OBPs are responsible for the detection of host plant attractants. A single OBP may participate in the recognition of several chemical odours (Vogt et al., 1991; Pelosi et al., 2014); however, our results reported that only OBP2 was significantly expressed in response to baits at each adult physiological stage among these 10 OBP genes. On the other hand, specific compounds might be recognized by more than one OBP gene, as the results of our study revealed that few OBP genes showed significant responses to spent brewer's yeast volatiles, while others showed no response. To the best of our knowledge, our data exhibit the relationship of these ten OBP genes to spent brewer's yeast volatiles, protein bait and irradiation for the first time.

The combination of different OBPs in insects may form a molecular basis for the ability to identify and distinguish relevant olfactory cues amid the vast spectrum of odorants emitted from fruits or plants (Takken and Knols, 1999; Zwiebel and Takken, 2004). In our study, some OBPs showed the same response to MIX treatment and were highly expressed at all physiological stages. This result further supports the view that OBPs are not just simple, general solubilizers that act as transporters for odorants but also play a vital role in the perception of different kinds of chemicals in the environment (Pelosi et al., 2006; Swarup et al., 2011). An OBP in Hylamorpha elegans showed a high binding affinity to $\beta$-ionone (Venthur et al., 2016), a volatile compound derived from plant carotenoids (Simkin et al., 2004).

Most of the OBP genes showed significant responses to MIX treatment at all adult life stages (Figure $4 a, 4 b$ and $4 c$ ). Irradiation has been reported to significantly destabilize fly fitness by damaging tissues and interior organs to some degree, inducing metabolic disorders (Lauzon and Potter, 2012; Yao et al., 2017), and our results showed that irradiation significantly altered the transcript levels of OBP genes at all adult life stages (Figure $5 a, 5 b$ and $5 c$ ), with especially abundant expression in the second and third stages (Figure 5b, 5c). These significant observations suggest that the effects of applied treatments on the transcript levels of BdorOBP genes may play an essential role in understanding the insect olfaction mechanism in response to different kinds of 
protein baits, volatiles released by different kinds of plants in the environment and irradiation applied to insects at the pupal stage.

Our results provide a direct BdorOBP transcript analysis that represents new evidence for the role of olfactory proteins in the reception of protein baits and volatiles. We believe that these findings establish a foundation for understanding the molecular mechanisms by which OBPs contribute to olfactory recognition and have applications for integrated pest management techniques. However, an urgent need exists to identi characterize the remaining ten $\mathrm{OBP}$ genes of $\mathrm{B}$. dorsalis in order to understand the transcriptional regulation of all OBPs in response to protein baits, volatiles and irradiation. The functional study of antennae-specific or antennae-enriched OBPs can assist us to better understand the involvement of OBPs in the mechanisms of insect olfaction with respect to different environmental stimuli and to design an odorant-based insect control strategy for the management of $B$. dorsalis.

\section{Conclusions}

Since its arrival in mainland China, $B$. dorsalis has become one of most destructive pests in orchards. Furthermore, this pest is presently spreading to more suitable areas of North China. The use of chemical insecticides remains the foremost way to control $B$. dorsalis in most developed countries, especially in China. In previous decades, various approaches have been established to monitor its detection and dispersal in order to minimize damage, and many studies have been carried out to interpret different aspects of its invasion mechanism. Further studies are still needed to understand its adaptation to new environments, resistance to chemicals, and high procreative ability. For the first time, we have evaluated the effects of different kinds of attractive baits, brewer's yeast volatiles and irradiation on the transcript expression levels of $10 \mathrm{OBP}$ genes in the antennae of B. dorsalis during three adult physiological stages. Some BdorOBP genes are significantly upregulated in response to the applied treatments, and others may play a vital role in mediating the response of males to irradiation, while OBP genes in females showed high transcript levels in response to different baits and brewer's yeast volatiles.

Acknowledgements. We express our appreciation to Ms Shumei Wang for rearing the B. dorsalis. This work was supported by the Institute of Beneficial Insects Laboratory, Fujian Agriculture and Forestry University, Fuzhou, Fujian Province, China. We sincerely thank Prof Lai Zhongxiong and Prof Zonghua Wang (Fujian Agriculture and Forestry University) for their kind help in allowing us to perform qRTPCR experiments in their Laboratory.

Conflicts of Interest: The authors declare no conflict of interest.

\section{REFERENCES}

[1] Chen, S. L., Dai, S. M., Lu, K. H., Chang, C. (2008): Female-specific doublesex dsRNA interrupts yolk protein gene expression and reproductive ability in oriental fruit fly, Bactrocera dorsalis (Hendel). - Insect Biochemistry and Molecular Biology 38: 155-165.

[2] Clarke, A. R., Armstrong, K. F., Carmichael, A. E., Milne, J. R., Raghu, S., Roderick, G. K., Yeates, D. K. (2005): Invasive phytophagous pests arising through a recent tropical evolutionary radiation: the Bactrocera dorsalis complex of fruit flies. - Annual Review of Entomology 50: 293-319. 
[3] Cornelius, M. L., Duan, J. J., Messing, R. H. (2000): Volatile host fruit odors as attractants for the oriental fruit fly (Diptera: Tephritidae). - Journal of Economic Entomology 93: 93-100.

[4] Dahanukar, A., Hallem, E. A., Carlson, J. R. (2005): Insect chemoreception. - Current Opinion in Neurobiology 15: 423-430.

[5] de Bruyne, M., Baker, T. C. (2008): Odor detection in insects: volatile codes. - Journal of Chemical Ecology 34: 882-897.

[6] Du, Y. J. Q., Chen, J., Lai, Z. (2015): Electroantennographic responses of Bactrocera dorsalis (Hendel), to volatile enzymatic hydrolysate from spent brewer's yeast. - Journal of Fujian Agriculture and Forestry University (Natural Science Edition) 444: 355-359.

[7] Du, Y., Zhenmin, L., Huijie, D. (2012): Analysis on Volatile Ingredients in Beer Waste Yeast Hydrolysate of Bactrocera dorsalis (Hendel) protein bait. - Journal of Anhui Agricultural Sciences 40: 3350.

[8] Finn, R. D., Coggill, P., Eberhardt, R. Y., Eddy, S. R., Mistry, J., Mitchell, A. L., Potter, S. C., Punta, M., Qureshi, M., Sangrador-Vegas, A., Salazar, G. A. (2016): The Pfam protein families database: towards a more sustainable future. - Nucleic acids research 44 : 279-285.

[9] Foret S., Maleszka, R. (2006): Function and evolution of a gene family encoding odorant binding-like proteins in a social insect, the honey bee (Apis mellifera). - Genome Research 16: 1404-1413. doi: 10.1101/gr.5075706

[10] Geib, S. M., Calla, B., Hall, B., Hou, S., Manoukis, N. C. (2014): Characterizing the developmental transcriptome of the oriental fruit fly, Bactrocera dorsalis (Diptera: Tephritidae) through comparative genomic analysis with Drosophila melanogaster utilizing modENCODE datasets. - BMC Genomics 15: 942.

[11] Hardy, D. E. (1973): The fruit flies (Tephritidae-Diptera) of Thailand and bordering countries. - Entomology Department, Bernice P. Bishop Museum, Honolulu.

[12] He, P., Zhang, J., Liu, N. Y., Zhang, Y. N., Yang, K., Dong, S. L. (2011): Distinct expression profiles and different functions of odorant binding proteins in Nilaparvata lugens Stål. - PLoS One 6: e28921.

[13] Hsu, J.-C., Chien, T.-Y., Hu, C.-C., Chen, M.-J. M., Wu, W.-J., Feng, H.-T., Haymer, D. S., Chen, C.-Y. (2012): Discovery of genes related to insecticide resistance in Bactrocera dorsalis by functional genomic analysis of a de novo assembled transcriptome. - PloS One 7: e40950.

[14] Hu, H., Wei, X., Cai, Z., Wu, R. (2007): Research and Application of Host Selection of Oriental Fruit Fly Bactrocera dorsalis. - Acta Agriculturae Jiangxi 19: 68-71.

[15] Hu, F., Dou, W., Wang, J. J., Jia, F. X., Wang, J. J. (2014): Multiple glutathione S-transferase genes: identification and expression in oriental fruit fly, Bactrocera dorsalis. - Pest management science 70: 295-303.

[16] Jang, E. B., Light, D. M. (1991): Behavioral responses of female oriental fruit flies to the odor of papayas at three ripeness stages in a laboratory flight tunnel (Diptera: Tephritidae). - Journal of Insect Behavior 4: 751-762.

[17] Jessup, A. J., Dominiak, B., Woods, B., De Lima, C. P. F., Tomkins, A., Smallridge, C. J. (2007): Area-wide management of fruit flies in Australia. - In: Vreysen, M. J. B., Robinson, A. S., Hendrichs, J. (eds.) Area-Wide Control of Insect Pests: from Research to Field Implementation. Springer Netherlands, Dordrecht, pp 685-697.

[18] Justice, R. W., Biessmann, H., Walter, M. F., Dimitratos, S. D., Woods, D. F. (2003): Genomics spawns novel approaches to mosquito control. - BioEssays 25: 1011-1020.

[19] Kumar, S., Stecher, G., Tamura, K. (2016): MEGA7: Molecular Evolutionary Genetics Analysis version 7.0 for bigger datasets. - Molecular biology and evolution 33: 18701874.

[20] Lauzon, C. R., Potter, S. E. (2012): Description of the irradiated and nonirradiated midgut of Ceratitis capitata Wiedemann (Diptera: Tephritidae) and Anastrepha ludens Loew 
(Diptera: Tephritidae) used for sterile insect technique. - Journal of pest science 85: 217226.

[21] Leal, W. S. (2013): Odorant reception in insects: roles of receptors, binding proteins, and degrading enzymes. - Annual Review of Entomology 58: 373-391.

[22] Leblanc, L., Vueti, E. T., Allwood, A. J. (2013): Host plant records for fruit flies (Diptera: Tephritidae: Dacini) in the Pacific Islands: 2. Infestation statistics on economic hosts. - Proceedings of the Hawaiian Entomological Society. Hawaiian Entomological Society 45: 83-117.

[23] Ling, C. (2013): Biological characteristics of Bactrocera dorsalis reared on different citrus fruits and mechanism of olfaction. - China Jiliang University.

[24] Liu, G., Huang, H., Liu, J., Ou, J., Wu, h., Zheng, J., Zhou, Z., Jian, J., Chen, Y. (2010): Attraction of volatiles from guava for Bactrocera dorsalis (Hendel). - Journal of Environmental Entomology 32: 291-294.

[25] Liu, N. Y., Yang, K., Liu, Y., Xu, W., Anderson, A., Dong, S. L. (2015): Two generalodorant binding proteins in spodoptera litura are differentially tuned to sex pheromones and plant odorants. - Comparative Biochemistry and Physiology. Part A, Molecular and Integrative Physiology 180: 23-31.

[26] Livak, K. J., Schmittgen, T. D. (2001): Analysis of relative gene expression data using real-time quantitative PCR and the 2- $\Delta \Delta C T$ method. - Methods 25: 402-408.

[27] Li, Q., Liberles, S. D. (2015): Aversion and attraction through olfaction. - Current Biology 25: 120-129. 10.1016/j.cub.2014.11.044

[28] Lou, Y. G., Ma, B., Cheng, J. A. (2005): Attraction of the parasitoid Anagrus nilaparvatae to rice volatiles induced by the rice brown planthopper Nilaparvata lugens. - Journal of Chemical Ecology 31: 2357-2372.

[29] Mau, R. F. L., Jang, E. B., Vargas, R. I. (2007): The Hawaii area-wide fruit fly pest management programme: influence of partnerships and a good education programme. In: Vreysen, M. J. B., Robinson, A. S., Hendrichs, J. (eds.) Area-Wide Control of Insect Pests: From Research to Field Implementation. Springer Netherlands, Dordrecht, 671683. Chapter: 62, doi: 10.1007/978-1-4020-6059-5_62

[30] Park, S. K., Shanbhag, S. R., Wang, Q., Hasan, G., Steinbrecht, R. A., Pikielny, C. W. (2000): Expression patterns of two putative odorant-binding proteins in the olfactory organs of Drosophila melanogaster have different implications for their functions. - Cell Tissue Research 300: 181-192. doi: 10.1007/s004410050059

[31] Papadopoulos, N. T., Plant, R. E., Carey, J. R. (2013): From trickle to flood: the largescale, cryptic invasion of California by tropical fruit flies. - Proceedings of the Royal Society B: Biological Sciences 280: 20131466.

[32] Pelosi, P., Mastrogiacomo, R., Iovinella, I., Tuccori, E., Persaud, K. C. (2014): Structure and biotechnological applications of odorant-binding proteins. - Applied Microbiology and Biotechnology 98: 61-70.

[33] Pelosi, P., Zhou, J. J., Ban, L. P., Calvello, M. (2006): Soluble proteins in insect chemical communication. - Cellular and Molecular Life Sciences 63: 1658-1676.

[34] Piñero, J. C., Mau, R. F. L., McQuate, G. T., Vargas, R. I. (2009a): Novel bait stations for attract-and-kill of pestiferous fruit flies. - Entomologia Experimentalis et Applicata 133: 208-216.

[35] Piñero, J. C., Mau, R. F. L., Vargas, R. I. (2009b): Managing oriental fruit fly (Diptera: Tephritidae), with Spinosad-based protein bait sprays and sanitation in papaya orchards in Hawaii. - Journal of Economic Entomology 102: 1123-1132.

[36] Qiao, H., Tuccori, E., He, X., Gazzano, A., Field, L., Zhou, J. J., Pelosi, P. (2009): Discrimination of alarm pheromone (E)- $\beta$-farnesene by aphid odorant-binding proteins. Insect Biochemistry and Molecular Biology 39: 414-419.

[37] Royer, J. E., De Faveri, S. G., Lowe, G. E., Wright, C. L. (2014): Cucumber volatile blend, a promising female-biased lure for Bactrocera cucumis (French 1907) (Diptera: 
Tephritidae: Dacinae), a pest fruit fly that does not respond to male attractants. - Austral Entomology 53: 347-352.

[38] Shen, G. M., Dou, W., Niu, J. Z., Jiang, H. B., Yang, W. J., Jia, F. X., Hu, F., Cong, L., Wang, J. J. (2011a): Transcriptome analysis of the oriental fruit fly (Bactrocera dorsalis). - PLOS One 6: e29127.

[39] Shen, J., Hu, L., Bin, S., Sun, X., Lin, J. (2011b): Attractive activity to Bactrocera dorsalis and chemical components analysis of volatiles from Musa supientum. - Journal of Zhongkai University of Agriculture and Engineering 24: 1-4.

[40] Siderhurst, M. S., Jang, E. B. (2010): Cucumber volatile blend attractive to female melon fly, Bactrocera Cucurbitae (Coquillett). - Journal of Chemical Ecology 36: 699-708.

[41] Simkin, A. J., Underwood, B. A., Auldridge, M., Loucas, H. M., Shibuya, K., Schmelz, E., Clark, D. G., Klee, H. J. (2004): Circadian regulation of the PhCCD1 carotenoid cleavage dioxygenase controls emission of $\beta$-ionone, a fragrance volatile of petunia flowers. - Plant Physiology 136: 3504-3514.

[42] Spencer, J., Fujita, B. (1998): A procedural manual for mass rearing four species of tephritid fruit flies. - United States Department of Agriculture, Agricultural Research Service, Honolulu, HI.

[43] SPSS for Windows (2007): SPSS for Windows, Version 16.0.

[44] Steinbrecht, R. A. (1997): Pore structures in insect olfactory sensilla: a review of data and concepts. - International Journal of Insect Morphology and Embryology 26: 229-245.

[45] Sun, L., Gu, S. H., Xiao, H. J., Zhou, J. J., Guo, Y. Y., Liu, Z. W., Zhang, Y. J. (2013a): The preferential binding of a sensory organ specific odorant binding protein of the alfalfa plant bug Adelphocoris lineolatus AlinOBP10 to biologically active host plant volatiles. Journal of Chemical Ecology 39: 1221-1231.

[46] Sun, L., Wei, Y., Zhang, D. D., Ma, X. Y., Xiao, Y., Zhang, Y. N., Yang, X. M., Xiao, Q., Guo, Y. Y., Zhang, Y. J. (2016a): The mouthparts enriched odorant binding protein 11 of the alfalfa plant bug Adelphocoris lineolatus displays a preferential binding behavior to host plant secondary metabolites. - Frontiers in Physiology 7: 201.

[47] Sun, L., Xiao, H. J., Gu, S. H., Zhou, J. J., Guo, Y. Y., Liu, Z. W., Zhang, Y. J. (2014): The antenna-specific odorant-binding protein AlinOBP13 of the alfalfa plant bug Adelphocoris lineolatus is expressed specifically in basiconic sensilla and has high binding affinity to terpenoids. - Insect Molecular Biology 23: 417-434.

[48] Sun, X., Zeng, F. F., Yan, M. J., Zhang, A., Lu, Z. X., Wang, M. Q. (2016b): Interactions of two odorant-binding proteins influence insect chemoreception. - Insect Molecular Biology 25: 712-723.

[49] Sun, X., Zhou, W., Liu, H., Zhang, A., Ai, C.-R., Zhou, S.-S., Zhou, C.-X., Wang, M.-Q. (2013b): Transgenic Bt rice does not challenge host preference of the target pest of rice leaffolder, Cnaphalocrocis medinalis (Lepidoptera: Pyralidae). - PloS One 8: e79032.

[50] Sun, Y. F., De Biasio, F., Qiao, H. L., Iovinella, I., Yang, S. X., Ling, Y., Riviello, L., Battaglia, D., Falabella, P., Yang, X. L. (2012): Two odorant-binding proteins mediate the behavioural response of aphids to the alarm pheromone (E)- $\beta$-farnesene and structural analogues. - PloS One 7: e32759.

[51] Swarup, S., Williams, T. I., Anholt, R. R. (2011): Functional dissection of Odorant binding protein genes in Drosophila melanogaster. - Genes, Brain, and Behavior 10: 648657.

[52] Takken, W., Knols, B. G. (1999): Odor-mediated behavior of Afrotropical malaria mosquitoes. - Annual Review of Entomology 44: 131-157.

[53] Tegoni, M., Campanacci, V., Cambillau, C. (2004): Structural aspects of sexual attraction and chemical communication in insects. - Trends in Biochemical Sciences 29: 257-264.

[54] Vargas, R. I., Piñero, J. C., Leblanc, L. (2015): An overview of pest species of Bactrocera fruit flies (Diptera: Tephritidae) and the integration of biopesticides with other biological approaches for their management with a focus on the Pacific region. - Insects 6: 297-318. 
[55] Venthur, H., Zhou, J., Mutis, A., Ceballos, R., Mella-Herrera, R., Larama, G., Avila, A., Iturriaga-Vásquez, P., Faundez-Parraguez, M., Alvear, M., Quiroz, A. (2016): $\beta$-Ionone as putative semiochemical suggested by ligand binding on an odorant-binding protein of Hylamorpha elegans and electroantennographic recordings. - Entomological Science 19: 188-200. doi: 10.1111/ens.12180.

[56] Vogt, R. G., Prestwich, G. D., Lerner, M. R. (1991): Odorant-binding-protein subfamilies associate with distinct classes of olfactory receptor neurons in insects. - Journal of Neurobiology 22: 74-84.

[57] Yao, M., Zhang, H., Cai, P., Gu, X., Wang, D., Ji, Q. (2017): Enhanced fitness of a Bactrocera cucurbitae genetic sexing strain based on the addition of gut-isolated probiotics (Enterobacter spec.) to the larval diet. - Entomologia Experimentalis et Applicata 162: 197-203.

[58] Yuan H. B., Ding Y. X., Gu S. H., Sun L., Zhu X. Q., Liu H. W., et al. (2015). Molecular characterization and expression profiling of odorant-binding proteins in Apolygus lucorum. - PLoS ONE10:e140562. doi: 10.1371/journal.pone.0140562

[59] Zhang, X., Jiang, Y., Peng, F., He, N., Li, Y., Zhao, D. (2007): Changes of Aroma Components in Hongdeng Sweet Cherry during fruit development. - Agricultural Sciences in China 6: 1376-1382. doi: 10.1016/S1671-2927(07)60186-2.

[60] Zheng, W., Peng, T., He, W., Zhang, H. (2012a): High-throughput sequencing to reveal genes involved in reproduction and development in Bactrocera dorsalis (Diptera: Tephritidae). - PloS One 7: e36463.

[61] Zheng, W., Peng, W., Zhu, C., Zhang, Q., Saccone, G., Zhang, H. (2013): Identification and Expression Profile Analysis of Odorant Binding Proteins in the Oriental Fruit Fly Bactrocera dorsalis. - International Journal of Molecular Sciences 14: 14936-14949.

[62] Zheng, W., Zhu, C., Peng, T., Zhang, H. (2012b): Odorant receptor co-receptor Orco is upregulated by methyl eugenol in male Bactrocera dorsalis (Diptera: Tephritidae). Journal of Insect Physiology 58: 1122-1127.

[63] Zhou, J.-J. (2010): Chapter ten - odorant-binding proteins in insects. - In: Litwack, G. (ed.) Vitamins \& Hormones. Academic Press, New York, 83: 241-272.

[64] Zwiebel, L. J., Takken, W. (2004): Olfactory regulation of mosquito-host interactions. Insect Biochemistry and Molecular Biology 34: 645-652. 